\title{
THE SINGULAR PART AS FIXED POINT
}

\author{
TAMÁS TITKOS
}

\begin{abstract}
This is an "Author's Original Manuscript" of an article published by
Taylor \& Francis Group

in The American Mathematical Monthly 125:1, 77-80.
\end{abstract}

Introduction. In his recent paper "On the mappings connected with parallel addition of nonnegative operators", Yu. M. Arlinskiu presented a very new approach to establish the Lebesgue type decomposition of bounded nonnegative operators (see $[2,3]$ ). The aim of this short note is to translate his approach to the case of nonnegative finite measures. Replacing the notion of parallel sum of bounded positive operators with the infimum of nonnegative finite measures we gain an elementary proof for the Lebesgue decomposition theorem. For other significantly different approaches see $[1,4,5,6,7]$.

Notions and notations. Let $\mathcal{A}$ be a $\sigma$-algebra of subsets of a nonempty set $X$. A $\sigma$-additive set function $\mu: \mathcal{A} \rightarrow \mathbb{R}_{+}$with $\mu(X)<+\infty$ is called shortly a measure. The identically zero measure will be denoted by $\theta$. Now, define absolute continuity and singularity as follows: $\mu$ is said to be $\nu$-absolutely continuous $(\mu \ll \nu$, in symbols) if $\nu(A)=0$ implies $\mu(A)=0$ for all $A \in \mathcal{A}$. Singularity of $\mu$ and $\nu$ (denoted by $\mu \perp \nu$ ) means that there exists a set $P \in \mathcal{A}$ such that $\mu(P)=\nu(X \backslash P)=0$.

Since singularity is the key notion of this paper, we present here the sketch of a well-known characterization. Namely, the following lemma states that singularity can be viewed as an order property. Recall first that the greatest lower bound of every two measures exists, and it can be expressed as follows

$$
(\mu \wedge \nu)(A)=\inf \{\mu(A \cap P)+\nu(A \backslash P) \mid P \in \mathcal{A}\} \quad \text { for all } A \in \mathcal{A} .
$$

Lemma. Let $\mu$ and $\nu$ be measures on $\mathcal{A}$. The greatest lower bound is the zero measure if and only if there exists a $P \in \mathcal{A}$ such that $\mu(P)=\nu(X \backslash P)=0$.

Proof. If such a $P \in \mathcal{A}$ exists, then $\vartheta \leq \mu$ and $\vartheta \leq \nu$ imply for all $A \in \mathcal{A}$ that

$$
\vartheta(A)=\vartheta(A \cap P)+\vartheta(A \backslash P) \leq \mu(P)+\nu(X \backslash P)=0 .
$$

For the converse implication assume that $\mu \wedge \nu$ is the zero measure. Then

$$
(\mu \wedge \nu)(X)=\inf \{\mu(X \cap P)+\nu(X \backslash P) \mid P \in \mathcal{A}\}=0
$$

implies for every $k \in \mathbb{N}$ that there exists a measurable subset $S_{k} \in \mathcal{A}$ such that

$$
\mu\left(X \cap S_{k}\right) \leq \frac{1}{2^{k+1}} \quad \text { and } \quad \nu\left(X \backslash S_{k}\right) \leq \frac{1}{2^{k+1}} .
$$

Now set $P_{n}:=\bigcup_{k=n}^{\infty} S_{k}$, then $P:=\bigcap_{n=1}^{\infty} P_{n}$ satisfies $\mu(P)=\nu(X \backslash P)=0$, because

$$
\mu\left(\bigcup_{k=n}^{\infty} S_{k}\right) \leq \frac{1}{2^{n}} \quad \text { and } \quad k \geq n \quad \text { implies } \quad \nu\left(X \backslash P_{n}\right) \leq \nu\left(X \backslash S_{k}\right) \leq \frac{1}{2^{k+1}}
$$


Now, we are going to prove the existence of the Lebesgue decomposition. Namely, we obtain the singular part as a fixed point of a standard iteration scheme, and than we show that the residual part is absolutely continuous. We do not deal with uniqueness, because it can be proved with the usual argument, independently from our approach.

According to the previous lemma, we can identify $\nu$-singular measures as fixed points of the measure valued map $\mathfrak{s}$ defined by

$$
\mathfrak{s}(\vartheta):=\vartheta-\vartheta \wedge \nu .
$$

Theorem. Let $\mu$ and $\nu$ be measures on the $\sigma$-algebra $\mathcal{A}$. Then $\mu$ has a decomposition $\mu=\mu_{a}+\mu_{s}$, where $\mu_{a} \ll \nu$ and $\mu_{s} \perp \nu$.

Proof. Observe first that $0 \leq \mathfrak{s}(\vartheta) \leq \vartheta$ for all $\vartheta$, and hence, the following iteration

$$
\mathfrak{s}^{[0]}(\mu):=\mu, \quad \text { and } \quad \mathfrak{s}^{[n]}(\mu):=\mathfrak{s}\left(\mathfrak{s}^{[n-1]}(\mu)\right) \quad \text { for } n \geq 1
$$

defines a decreasing sequence of measures. Let denote the pointwise limit (which is a measure as well) with $\mu_{s}$. Also, observe the following two consequences of (1) and (2)

$$
\mathfrak{s}^{[n]}(\mu) \wedge \nu=\mathfrak{s}^{[n]}(\mu)-\mathfrak{s}^{[n+1]}(\mu) \quad \text { and } \quad \mu-\mathfrak{s}^{[n+1]}(\mu)=\sum_{k=1}^{n}\left(\mathfrak{s}^{[n]}(\mu) \wedge \nu\right) .
$$

The first formula implies that $\mu_{s}$ and $\nu$ are singular, the second one guarantees the $\nu$-absolute continuity of $\mu_{a}:=\mu-\mu_{s}$. Indeed, $\mu_{s} \wedge \nu=\theta$ according to

$$
\mu_{s} \wedge \nu=\lim _{n \rightarrow \infty}\left(\mathfrak{s}^{[n]}(\mu) \wedge \nu\right)=\lim _{n \rightarrow \infty}\left(\left(\mathfrak{s}^{[n]}(\mu)-\mu_{s}\right)-\left(\mathfrak{s}^{[n+1]}(\mu)-\mu_{s}\right)\right)=\theta,
$$

and if $A \in \mathcal{A}$ such that $\nu(A)=0$, then $\left(\mathfrak{s}^{[k]}(\mu) \wedge \nu\right)(A)=0$ for all $k \in \mathbb{N}$, hence

$$
\mu_{a}(A)=\left(\lim _{n \rightarrow \infty}\left(\mu-\mathfrak{s}^{[n+1]}(\mu)\right)\right)(A)=\lim _{n \rightarrow \infty} \sum_{k=0}^{n}\left(\mathfrak{s}^{[k]}(\mu) \wedge \nu\right)(A)=0 .
$$

Acknowledgment. The author was supported by the National Research, Development and Innovation Office, NKFIH-104206.

\section{REFERENCES}

[1] C. D. Aliprantis, O. Burkinshaw, Principles of Real Analysis, Third Edition, Academic Press, Inc., San Diego, CA, 1998.

[2] T. Ando, Lebesgue-type decomposition positive operators, Acta Scientiarum Mathematicarum (Szeged), 38 (1976), 253-260.

[3] Yu. M. Arlinskiĭ, On the mappings connected with parallel addition of nonnegative operators, Positivity, 21 (2017), 299-327.

[4] S. K. Brooks The Lebesgue decomposition theorem for measures, The American Mathematical Monthly, 78 (1971), 660-662.

[5] A. R. Schep, And still one more proof of the Radon-Nikodym theorem, The American Mathematical Monthly 110 (2003), 526-538.

[6] Zs. Tarcsay, A functional analytic proof of the Lebesgue-Darst decomposition theorem, Real Analysis Exchange, 39 (2013), 219-226.

[7] T. Titkos, A simple proof of the Lebesgue decomposition theorem, The American Mathematical Monthly, 122 (2015), 793-794.

Tamás Titkos, Alfréd Rényi Institute of Mathematics, Hungarian Academy of Sciences, Reáltanoda u. 13-15., Budapest H-1053, Hungary

E-mail address: titkos.tamas@renyi.mta.hu 